\title{
ARTICLE OPEN Multi-time quantum correlations with no spatial analog
}

\author{
Martin Ringbauer $\mathbb{D}^{1,2,3}$, Fabio Costa ${ }^{2}$, Michael E. Goggin ${ }^{2,4}$, Andrew G. White $\mathbb{D}^{2}$ and Alessandro Fedrizzi $\mathbb{D}^{1}$
}

Multipartite quantum correlations are a powerful resource that underpins applications from quantum metrology to quantum computing. While most research has focused on spatial correlations, it is now becoming clear that a sequence of measurements on a single quantum system at different points in time reveals a similarly rich, yet fundamentally different structure of multipartite temporal correlations. Here we experimentally observe genuine multi-time correlations in a sequence of three generalized measurements on a single photon. These correlations, manifest by a simultaneous violation of all pairwise Bell inequalities, cannot be reproduced by any spatial quantum state of equal dimension. Our work lays the foundation for the exploration of temporal correlations arising in quantum networks for quantum information applications.

npj Quantum Information (2018)4:37; doi:10.1038/s41534-018-0086-y

\section{INTRODUCTION}

First studied in the context of macroscopic realism, ${ }^{1}$ temporal quantum correlations have become of increasing interest for fundamental questions, ${ }^{2}$ and quantum information applications. ${ }^{3-}$ 6 Beyond the simplest scenarios, however, the equivalence between spatial and temporal correlations breaks down, revealing a rich structure of correlations in either domain. Comparing the resources generating these correlations shows that temporal processes cannot mimic every spatial quantum state, while spatial measurements are more restricted than their temporal counterpart. $^{7}$ Spatial correlations in this regime, in particular genuine multipartite entanglement, have become an important resource for foundational questions as well as quantum technologies such as quantum metrology and quantum computing.

Here we study multi-time correlations between three parties and observe a form of temporal correlations that cannot be reproduced by any multipartite quantum state of equal dimension. These correlations, which are revealed by temporal measurements that have no spatial analog, manifest in the simultaneous violation of pairwise Bell inequalities between all pairs of parties. This is in stark contrast to one of the fundamental features of spatial correlations-monogamy of entanglement. ${ }^{8,9}$ The fact that in the spatial scenario, Alice can either violate a Bell inequality with Bob, or with Charlie, but not both at the same time, is the basis for the security of entanglement-based quantum key distribution. Despite being polygamous in this sense, however, we show that multi-time correlations are still bound by a new polygamy relation that we derive.

\section{RESULTS}

Monogamy of entanglement

Consider first the scenario introduced by John Bell, ${ }^{10}$ where two space-like separated observers, Alice and Bob, perform local measurements on the parts of a shared state of two entangled two-level systems (qubits) (Fig. 1a). All correlations arising from classical systems and shared randomness in such a scenario must satisfy the Clauser-Horne-Shimony-Holt $(\mathrm{CHSH})^{11}$ inequality,

$S_{\mathrm{CHSH}}^{\mathrm{AB}}=\left\langle A_{0} B_{0}\right\rangle-\left\langle A_{0} B_{1}\right\rangle+\left\langle A_{1} B_{0}\right\rangle+\left\langle A_{1} B_{1}\right\rangle \leq 2$,

where $\left\langle A_{x} B_{y}\right\rangle=\sum_{a, b} a b P(a, b \mid x, y)$ denotes the joint expectation value for Alice's and Bob's measurements for settings $x$ and $y$ and outcomes $a$ and $b$, respectively. This inequality can be derived from the assumptions of realism, free choice, and no fine-tuning, which implies that if there is no observable signaling between two variables, then there should also be no hidden signaling in the underlying reality. Correlations obtained from entangled quantum states, however, can violate the $\mathrm{CHSH}$ inequality, indicating that they cannot be reproduced by any classical model without resorting to additional causal influences which are carefully hidden from the observable statistics. Spatial quantum correlations that violate a CHSH inequality are then commonly referred to as Bell-nonlocal.

One of the distinctive features of spatial Bell-nonlocal correlations $^{12}$ is that a violation of $S_{\mathrm{CHSH}}^{\mathrm{AB}}$ precludes a simultaneous violation of the $\mathrm{CHSH}$ inequality with a third space-like separated party, Charlie. This is a consequence of monogamy of entanglement, ${ }^{8,9}$ and is formally captured by the inequality, ${ }^{13}$

$S_{\mathrm{CHSH}}^{\mathrm{AB}}+S_{\mathrm{CHSH}}^{\mathrm{BC}} \leq 4$,

and similarly for the combinations $(A B, A C)$ and $(A C, B C)$. Simply put, for a given set of measurements, Bob's quantum system can only violate a CHSH inequality with Alice's system or Charlie's system, but not both at the same time (see Fig. 1c). This is in stark contrast to classical correlations, which can be shared arbitrarily, and underpins the security of entanglement-based quantum key distribution. ${ }^{14}$

In the corresponding temporal scenario, Fig. 1b, where Alice and Bob perform unbiased measurements on a single quantum system at different times, an inequality formally equivalent to the spatial CHSH inequality, Eq. (1), holds between each pair of parties. $^{3,7}$ In contrast to the spatial case, however, temporal

\footnotetext{
${ }^{1}$ Institute of Photonics and Quantum Sciences, School of Engineering and Physical Sciences, Heriot-Watt University, Edinburgh EH14 4AS, UK; ${ }^{2}$ Centre for Engineered Quantum Systems, School of Mathematics and Physics, University of Queensland, Brisbane, QLD 4072, Australia; ${ }^{3}$ Institut für Experimentalphysik, Universität Innsbruck, Innsbruck 6020, Austria and ${ }^{4}$ Department of Physics, Truman State University, Kirksville, MO 63501, USA

Correspondence: Alessandro Fedrizzi (a.fedrizzi@hw.ac.uk)
}

Received: 23 January 2018 Revised: 27 June 2018 Accepted: 4 July 2018

Published online: 02 August 2018 

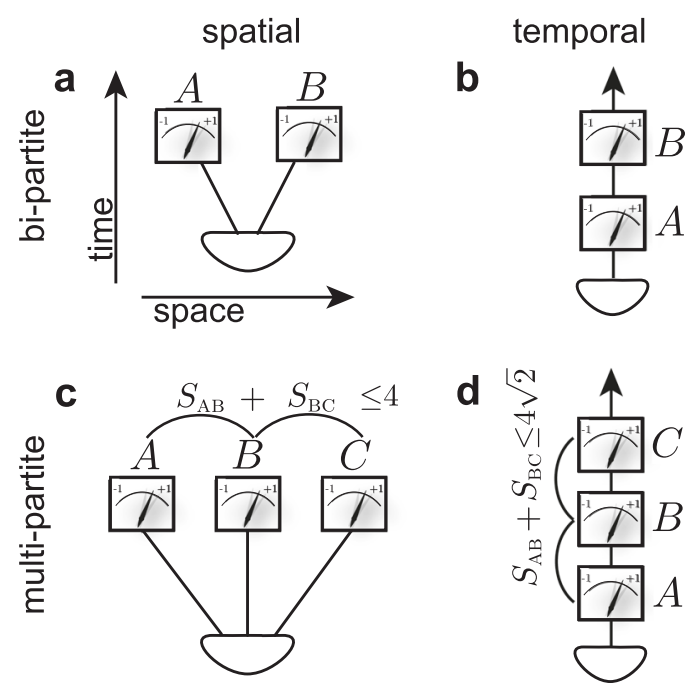

Fig. 1 Quantum correlations in space and time. a In typical Bell scenarios, Alice $(A)$ and Bob $(B)$, perform space-like separated measurements on parts of a shared entangled state $\left|\phi^{+}\right\rangle$. $\mathbf{b}$ In the corresponding temporal scenario, $A$ and $B$ measure the same quantum system at different times. In both cases, quantum correlations can violate a CHSH inequality. c For three parties, Alice, Bob, and Charlie $(C)$, monogamy of entanglement precludes a simultaneous spatial CHSH inequality violation between $A$ and $B$ and $B$ and $C$, respectively. d In the temporal scenario, monogamy of entanglement can be maximally violated: both, $S_{\mathrm{CHSH}}^{\mathrm{AB}}$ and $S_{\mathrm{CHSH}}^{\mathrm{BC}} \mathrm{can}$ simultaneously saturate the quantum bound

correlations are not bound to be monogamous. Instead, in a series of three projective measurements (see Fig. 1d), the monogamy condition of Eq. (2) can be violated up to a value of $4 \sqrt{2}$, showing that Bob can at the same time maximally violate the $\mathrm{CHSH}$ inequality with Alice and with Charlie.

\section{Formalizing tripartite temporal correlations}

To see this, consider a sequence of measurements $\mathcal{M}_{i}$ on a single quantum system in the initial state $\rho_{0}$, which undergoes Markovian, i.e., memoryless, evolution. To formalize this, we will use the "process matrix" formalism ${ }^{15}$ in the rest of the manuscript, following the treatment of ref. [7]. There is a number of related formalisms for temporal correlations that would lead to a similar analysis, up to some differences in conventions and emphasis. ${ }^{16-}$ 20 The crucial feature of these formalisms is that to each instant in time where a measurement can be performed, a double Hilbert space is associated, representing the system just before and just after the measurement. Approaches that assign a single Hilbert space per instant in time also exist, ${ }^{21-23}$ but face severe limitations ${ }^{24}$ and are unsuitable for the multipartite scenario in which we are interested.

Formally, the evolution of the system between the measurements is described by completely positive trace-preserving (CPTP) maps $\mathcal{T}_{j}$, while the measurement with settings $x$ and outcomes $a$ is in general described by an instrument. ${ }^{25} \mathrm{An}$ instrument $\left\{\mathcal{M}_{a \mid x}\right\}_{a}$ is a collection of completely positive, trace nonincreasing maps $\mathcal{M}_{a \mid x}: A_{l} \mapsto A_{O}$ from the input Hilbert space $A_{l}$ representing the system before the measurement, to the output space $A_{O}$ of the system after the measurement, such that $\sum_{a} \mathcal{M}_{a \mid x}$ is CPTP. Following ref. [7] the joint probability distribution for outcomes $a, b, c$, in a sequence of three measurements with settings $x, y, z$ can then be written as:

$$
\begin{aligned}
P(a, b, c \mid x, y, z)=\operatorname{tr} & {\left[\left(M_{a \mid x}^{A_{1} A_{0}} \otimes M_{b \mid x}^{B_{1} B_{0}} \otimes E_{c \mid z}^{C_{1}}\right) \cdot W^{A_{l} A_{o} B_{1} B_{0} C_{1}}\right] } \\
& W^{A_{1} A_{0} B_{1} B_{0} C_{1}}=\rho_{0}^{A_{1}} \otimes T_{1}^{A_{0} B_{1}} \otimes T_{2}^{B_{0} C_{1}}
\end{aligned}
$$

where $W^{A_{1} A_{0} B_{l} B_{O} C_{1}}$ is the so-called process matrix, ${ }^{15}$ which represents the resource shared by the three temporally separated parties. The matrices $M_{a \mid x}^{A_{1} A_{O}}$ and $T_{1}^{A_{0} B_{1}}$ are the Choi-Jamiołkowski representation of $\mathcal{M}_{a \mid x}$ and $\mathcal{T}_{1}$, respectively. ${ }^{7}$

The general form of $W$ in Eq. (3) and the possible correlations $P$ $(a, b, c \mid x, y, z)$ arise from the following assumptions: first, causality, together with temporal ordering, requires that past outcomes cannot depend on future settings. This is ensured by the fact that operations are described by quantum instruments, and that $W$ satisfies the conditions of a quantum comb, ${ }^{26,27}$ i.e., that it can be reproduced as a quantum circuit. Second, we assume that the process is memoryless, which means that $W$ has to obey the quantum Markov condition, ${ }^{20,28}$ leading to the product form of Eq. (3). Note also that, since the system is discarded after the final measurement, the output space $C_{O}$ becomes trivial and Charlie's instrument $\left\{M_{c \mid z}^{C_{1} C_{0}}\right\}_{c}$ reduces to a positive operator-valued measure (POVM) with elements $\left\{E_{c \mid z}^{C_{l}}\right\}_{c}$.

In the case where Alice, Bob, and Charlie perform temporally separated measurements on a single qubit, which is initially in a maximally mixed state and undergoes trivial evolution between each measurement, the process matrix in Eq. (3) is given by:

$W^{A_{0} B_{1} B_{0} C_{1}}=[[1]]^{A_{0} B_{1}} \otimes[[1]]^{B_{0} C_{1}}$,

where $[[1]]^{X Y}:=\sum_{j l}|j\rangle\left\langle\left. I\right|^{X} \otimes \mid j\right\rangle\left\langle\left. I\right|^{Y}\right.$ represents the identity map between Hilbert spaces $X$ and $Y$. Note that we have discarded the initial maximally mixed state by replacing Alice's measurement $M_{a \mid x}^{A_{1} A_{O}}$ with the preparation of a state $\left(\rho_{a \mid x}^{A_{O}}\right)^{T} / \operatorname{tr} \rho_{a \mid x}^{A_{O}}$ with probability $P(a \mid x)=\operatorname{tr} \rho_{a \mid x^{\prime}}^{A_{O}}$ where $\rho_{a \mid x}^{A_{O}}=\operatorname{tr}_{A_{l}} M_{a \mid x}^{A_{1} A_{O}}$.

Within this framework it is now straightforward to compare temporal to spatial resources for quantum correlations. ${ }^{7}$ In this context, the "spatial" character of the resource is defined by the requirement of no-signaling among the parties, without a direct reference to their spatio-temporal location. The process matrix of a no-signaling process only contains input spaces, on which only local POVM measurements can be performed. In other words, a non-signaling process is an ordinary multipartite state (output spaces equal to the identity matrix can also be added to a nonsignaling process, but without any effect on the generated correlations).

Specifically, since every process matrix is a valid quantum state, the spatial resource associated with the temporal process matrix of Eq. (4) is simply the spatial quantum state $|\psi\rangle^{A B C}=\left|\phi^{+}\right\rangle^{A B_{1}}\left|\phi^{+}\right\rangle^{B_{2} C}$, where $\left|\phi^{+}\right\rangle=\sum_{j}|j\rangle \otimes|j\rangle$ is a maximally entangled state. Note that, since Bob's temporal measurement acts on two Hilbert spaces, in the spatial analog Bob has to receive two systems, $B \equiv B_{1} \otimes B_{2}$, while Alice and Charlie receive one each. This implies that the correlations obtainable in a qubit entanglement-swapping scenario ${ }^{29}$ can also be obtained in a sequence of three projective temporal measurements (see Fig. 2). However, the converse is not true: the entanglement-swapping scenario cannot generate all the three-party temporal correlations. For example, reproducing the temporal violation of the monogamy condition, Eq. (2), in the spatial entanglement-swapping configuration would require Bob to postselect on equal outcomes on his qubits. Consequently, a violation is not possible by exploiting the spatial resource alone.

In general, only the first (Alice) and last (Charlie) measurements in the above scenario have a spatial analog, since these measurements have a trivial input and output Hilbert space, respectively. In contrast, Bob's intermediate measurement $M_{b \mid y}^{B_{1} B_{O}}$ can be more general and may not have a spatial analog. ${ }^{5,7}$ For example, the operation $M^{B_{1} B_{O}}=[[1]]^{B_{1} B_{0}}$, where $B$ leaves the system unperturbed in the temporal scenario, is a CPTP map that can be 


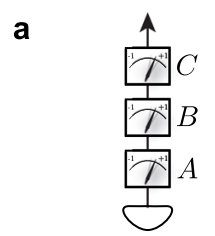

b

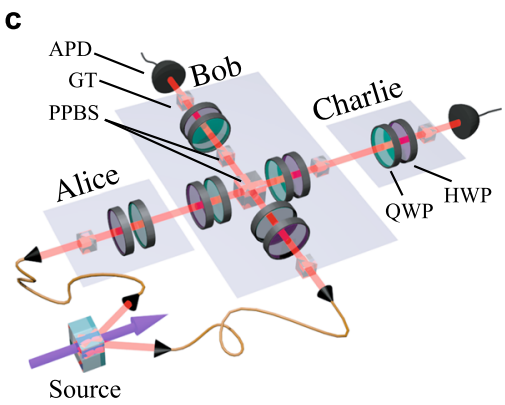

Fig. 2 Experimental test of tripartite temporal correlations. a $A, B$, and $C$ perform temporally separated measurements on a qubit. $\mathbf{b}$ The corresponding spatial correlation scenario resembles an entanglement-swapping configuration, where Alice and Charlie receive one particle, while Bob receives two. c Experimental setup implementing tripartite temporal correlations with weak measurements. Pairs of single photons are produced via spontaneous parametric downconversion in a $\beta$-barium borate crystal. One of these photons acts as the system on which we perform measurements at three times. The initial preparation $(A)$ and final measurement $(C)$ of the system photon are projective, while the intermediate measurement $(B)$ is non-projective. The latter is realized by partially entangling system and meter photons using a non-deterministic controlled-NOT gate, based on non-classical interference in a partially polarizing beam splitter. ${ }^{31}$ The measurement strength $\kappa$ can be continuously controlled via the state of the meter photon

performed with unit probability, whereas the corresponding POVM element in the spatial scenario, $E^{B_{1} B_{2}}=\left|\phi^{+}\right\rangle\left\langle\left.\phi^{+}\right|^{B_{1} B_{2}}\right.$, is a Bell-state measurement that can be implemented only with probability $1 / 4 .^{30}$

A crucial consequence of this observation, as we will show below, is that Alice, Bob, and Charlie can use the same correlation resource more effectively in the temporal case than in the spatial case. Specifically, the process matrix of Eq. (4) is equivalent to a pair of spatially separated Bell states, yet it gives rise to temporal correlations that cannot be reproduced in the spatial setting with systems of equal dimension.

\section{Experimental results}

Experimentally we study tripartite temporal correlations on photonic qubits, using the setup in Fig. 2c. As discussed above, the first and last measurements in such a multipartite temporal correlations experiment can be projective, while the intermediate measurements must be non-projective in order to reveal genuine multipartite temporal correlations, ${ }^{5,7}$ which cannot be decomposed into bipartite correlations. Alice and Charlie thus perform standard projective polarization measurements (note that Alice's preparation is equivalent to a measurement up to a transpose of the transformation operator) on the system photon, while Bob performs a variable-strength polarization measurement. The latter is implemented using a non-deterministic controlled-not (CNOT) gate with the system as the control qubit and a meter photon as the target qubit in the state $|\psi\rangle_{m}=\sqrt{\frac{1+\kappa}{2}}|0\rangle+\sqrt{\frac{1-\kappa}{2}}|1\rangle$, where $\kappa$ determines the measurement strength ( $k=1$ corresponds to a projective measurement; $K=0$ leaves the system unperturbed). A measurement of the meter photon in the computational basis $\{|0\rangle,|1\rangle\}$, together with appropriate unitary rotations of the system qubit before and after the interaction, implements a measurement of the system in an arbitrary basis ${ }^{31}$ (note that Alice's preparation is equivalent to a measurement up to a transpose of the transformation operator) ${ }^{32,33}$ with an average measurement fidelity of $\mathcal{F}_{\mathrm{B}}=0.995_{-0.002}^{+0.002}$ ( $3 \sigma$ uncertainty regions) for small $\kappa$ and $\mathcal{F}_{\mathrm{B}}=0.983_{-0.01}^{+0.01}$ for large $\kappa$. This fidelity is primarily limited by the fidelity of the CNOT gate, and the projective measurements for Alice and Charlie achieve fidelities of $\mathcal{F}_{\mathrm{A}}=$ $0.9991_{-0.0009}^{+0.0006}$ and $\mathcal{F}_{\mathrm{C}}=0.9992_{-0.0009}^{+0.0006}$, respectively.

To test the temporal CHSH inequality (1), we choose measurement settings in the $x z$-plane of the Bloch sphere as $A_{0,1}=$ $\cos \phi_{A} \hat{X} \pm \sin \phi_{A} \hat{Z}$ for Alice, and similarly for Bob and Charlie (see Fig. 3a). For $K \sim 1$, where all measurements are projective, and with $\phi_{\mathrm{A}}=0$ and $2 \phi_{\mathrm{B}}=\phi_{\mathrm{C}}=\phi$, we observe a simultaneous violation of the temporal CHSH inequality (1) for Alice-Bob, and Bob-Charlie, of up to $S_{\mathrm{CHSH}}^{\mathrm{AB}} \sim S_{\mathrm{CHSH}}^{\mathrm{BC}}=2.74_{-0.03}^{+0.03}$. All quoted uncertainties correspond to $3 \sigma$-equivalent statistical confidence regions obtained from Monte-Carlo resampling according to Poissonian counting statistics. These results clearly indicate the presence of temporal entanglement and also demonstrate a violation of the monogamy condition (2) for almost the full range of $0<\phi<\pi$ (see Fig. 3c). At the same time we note that the observed correlations factorize into bipartite correlations, as indicated by the total variation between $P(a, b, c \mid x, y, z)$ and $P(a, b \mid x, y) P(b, c \mid y, z)$ of $0.027_{-0.003}^{+0.005}$. Furthermore, the CHSH inequality between $A$ and $C$ remains unviolated with a value of $S_{\mathrm{CHSH}}^{\mathrm{AC}}=1.05_{-0.03}^{+0.03}$.

More surprising results emerge when we consider weak measurements for Bob. For measurement strengths $0.7 \lesssim \kappa \lesssim$ 0.92 and $4 \pi / 32 \lesssim \phi \lesssim 14 \pi / 32$, we observe a simultaneous violation of the CHSH inequality for all pairs $A B, B C$, and $A C$ (see Fig. 3b). For nominal values of $K=0.825$ and $\phi=5 \pi / 16$, we obtain simultaneous values of up to $S_{\mathrm{CHSH}}^{\mathrm{AB}}=2.25_{-0.03}^{+0.03}, S_{\mathrm{CHSH}}^{\mathrm{BC}}=2.18_{-0.03}^{+0.03}$, and $S_{\mathrm{CHSH}}^{\mathrm{AC}}=2.14_{-0.03}^{+0.03}$ (see Fig. $3 \mathrm{~d}$ ). Note that all operations performed by the three parties are uncorrelated and unbiased in the sense of ref. 7 and further satisfy no-signaling between every pair. Therefore, as discussed in ref. [7], a corresponding classical resource should obey the $\mathrm{CHSH}$ inequalities, proving the genuine quantum nature of the observed correlations.

Importantly, this simultaneous violation of all pairwise temporal $\mathrm{CHSH}$ inequalities demonstrates the presence of a hitherto unknown form of temporal quantum correlations that cannot be reproduced in a spatial scenario of equal dimension. This disconnect is a direct consequence of the larger set of quantum measurements available in the temporal case. Conversely, it is known that there are multipartite spatial quantum states, such as W states, that lead to statistics that cannot be reproduced by a temporal process of equal dimension. ${ }^{7}$

\section{Limits of tripartite temporal correlations}

Curiously, in contrast to the usual monogamy of entanglement in Eq. (2), genuine multipartite temporal correlations, even with arbitrary generalized measurements, cannot achieve the algebraic maximum joint violation of the three pairwise $\mathrm{CHSH}$ inequalities between Alice, Bob, and Charlie for a single-qubit Markov process. Indeed, we show in the Methods that the maximal simultaneous $\mathrm{CHSH}$ value $S_{\max }$ for three pairs $A B, B C$, and $A C$ is bounded by:

$S_{\max } \approx 2.426$,

strictly below the algebraic maximal joint violation of $S_{\max }=10 /$ $3 \approx 3.33$. An important consequence of this observation is that the set of temporal quantum correlations has a non-trivial structure, since not all logically valid correlations can be obtained.

\section{DISCUSSION}

In the above experiments, we have verified the genuine multipartite nature of the observed correlations through the simultaneous violation of all pairwise temporal $\mathrm{CHSH}$ inequalities. While this rules out an explanation in terms of bipartite temporal correlations, it would be desirable to develop a more specific, quantitative measure for such genuine multipartite temporal 
a

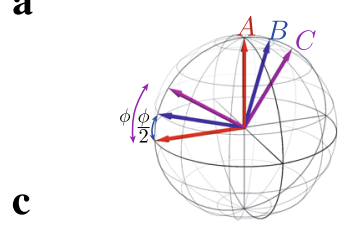

2.8

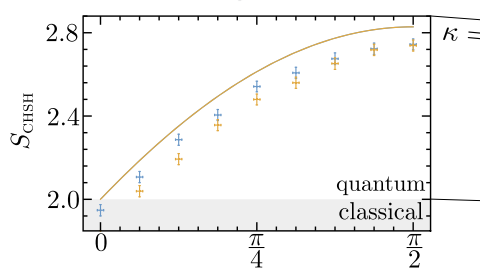

b

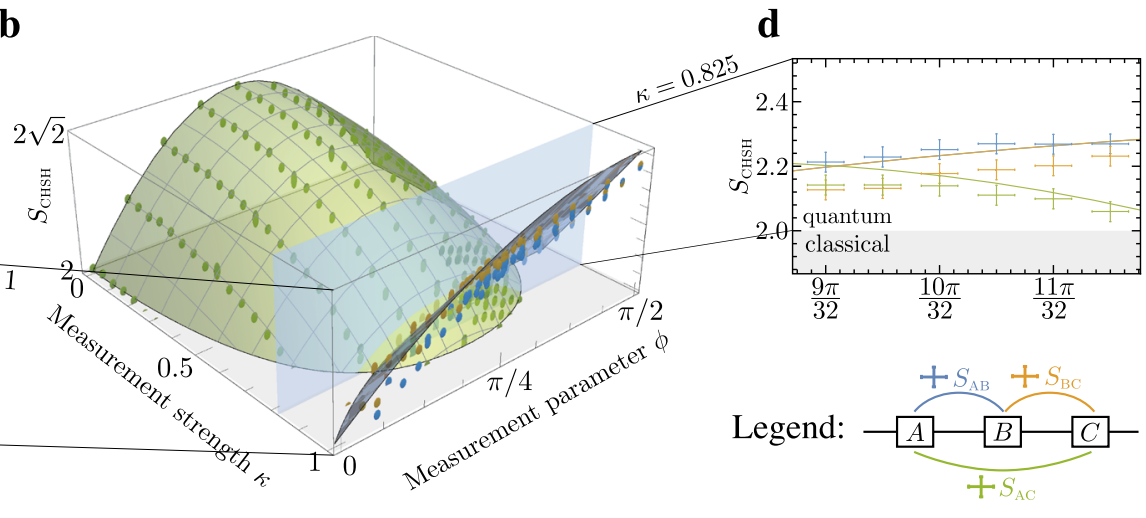

Fig. 3 Multipartite temporal CHSH correlations. a Measurement settings illustrated on the Bloch sphere. Alice's measurements are fixed and Bob's measurements enclose an angle $\phi / 2$ with Alice and Charlie's measurements. b Experimental CHSH values $S_{C H S H}^{A B}$ (blue), $S_{C H S H}^{B C}$ (orange), and $S_{C H S H}^{A C}$ (green), for $A B, B C$, and $A C$, respectively, vs. the weak measurement strength, $k$, and $\phi(a)$. The vertical axis is truncated at $S=2$, to highlight the non-classical aspect, and the 3D-ellipsoidal datapoints represent $3 \sigma$ experimental uncertainties. Error bars for $S_{\text {CHSH }}$ are $3 \sigma$ statistical uncertainties due to Poissonian counting statistics, while the error bars for $K$ and $\phi$ correspond to $3 \sigma$ confidence regions estimated from device calibration. c For $K=1$ all parties perform projective measurement, yielding maximal violation of the monogamy condition, Eq. (2), for $A B$ and $B C$, while the correlations between $A C$ remain classical. $\mathbf{d}$ A cut through the surfaces panel $\mathbf{b}$ at $k=0.825$. All data points are above the classical bound $S=2$

correlations. Such measures exist for spatial multipartite correlations, ${ }^{34}$ e.g., the residual tangle. However, they are insufficient for characterizing the correlations studied here. The reason for this shortcoming is that these measures charcterize the correlations that can be extracted from a given resource by means of local POVMs. In contrast, more general operations are available in the temporal case, which can extract correlations, such as those in Fig. $3 \mathrm{~d}$ from a given resource that are inaccessible in the spatial case. It would further be interesting to attempt a geometric characterization of tripartite or more general multipartite temporal correlations as has been done for bipartite correlations. ${ }^{23}$

In parallel with the study of temporal correlations, a particularly intriguing question concerns the non-classical nature of multipartite temporal correlations. ${ }^{5,35,36}$ Since all correlations obtained from the experiment in Fig. $2 c$ obey the no-signaling constraints between all pairs of parties, one can invoke the no fine-tuning principle of ref. [7] and derive inequalities that must be satisfied by a corresponding classical process. A related approach is to find a classical causal model that satisfies the same conditional independencies as in the experiment. ${ }^{36}$ However, the probabilities arising from such models form a non-convex set, which is very challenging to characterize already in the simplest cases. Establishing the non-classicality of these correlations has important implications for temporal quantum communication protocols, $^{3,37}$ temporal quantum computing, ${ }^{5}$ and future quantum networks, which inevitably feature both spatial and temporal correlations. ${ }^{2,38,39}$

\section{METHODS}

Theoretical expectation values

To understand the strong violation of monogamy of Bell-nonlocality observed in Fig. 3, consider three temporally separated measurements on a single qubit, a strong measurement $A$, followed by a weak measurement $B$, and a final strong measurement $C$ (with trivial evolution between the measurements), as illustrated in Fig. 2a. The Choi representation of a weak measurement in the computational basis, yielding outcome $b= \pm 1$, is:

$\left.\left|Q_{b}\right\rangle\right\rangle^{B_{1} B_{O}}=\sqrt{\frac{1+\kappa}{2}}|b\rangle^{B_{1}}|b\rangle^{B_{O}}+\sqrt{\frac{1-\kappa}{2}}|-b\rangle^{B_{1}}|-b\rangle^{B_{O}}$, a measurement in a different basis, obtained by rotating the qubit with a unitary $U$, is given by $\left.U^{T} \otimes U^{\dagger}\left|Q_{b}\right\rangle\right\rangle^{B_{B} B_{O}}$. For two measurements along directions $\boldsymbol{\alpha}$ and $\boldsymbol{\beta}$ on the Bloch sphere, the bipartite correlations are given by:

$$
\langle A B\rangle:=\sum_{a b} a b P(a, b \mid \mathbf{a}, \boldsymbol{\beta}) .
$$

When $A, B$, and $C$ perform measurements along direction $\boldsymbol{a}, \boldsymbol{\beta}$, and $\boldsymbol{\gamma}$, respectively, then the correlations are given by:

$$
\begin{aligned}
& \langle A B\rangle=\sqrt{\frac{1-\kappa}{2}} \boldsymbol{a} \cdot \boldsymbol{\beta}, \\
& \langle A C\rangle=\sqrt{\frac{1+\kappa}{2}} \boldsymbol{\alpha} \cdot \boldsymbol{\gamma}+\left(1-\sqrt{\frac{1+\kappa}{2}}\right) \boldsymbol{a} \cdot \boldsymbol{\beta} \boldsymbol{\gamma} \cdot \boldsymbol{\beta}, \\
& \langle B C\rangle=\sqrt{\frac{1-\kappa}{2}} \boldsymbol{\beta} \cdot \boldsymbol{\gamma} .
\end{aligned}
$$

For the CHSH quantity $S_{C H S H}^{A B}=\left\langle A_{0} B_{0}\right\rangle-\left\langle A_{0} B_{1}\right\rangle+\left\langle A_{1} B_{0}\right\rangle+\left\langle A_{1} B_{1}\right\rangle$ it follows that:

$S^{\mathrm{AB}}=\sqrt{\frac{1-\kappa}{2}} S_{\text {strong }}$

$S_{\beta}^{\mathrm{AC}}=\sqrt{\frac{1+\kappa}{2}} S_{\text {strong }}+\left(1-\sqrt{\frac{1+\kappa}{2}}\right) S_{\text {deph } \beta}$,

$S^{B C}=\sqrt{\frac{1-\kappa}{2}} S_{\text {strong }}$,

where

$S_{\text {strong }}=\boldsymbol{a}_{0} \cdot\left(\boldsymbol{\beta}_{0}-\boldsymbol{\beta}_{1}\right)+\boldsymbol{a}_{1} \cdot\left(\boldsymbol{\beta}_{0}+\boldsymbol{\beta}_{1}\right)$

is the $\mathrm{CHSH}$ value for parties performing strong measurements with settings $\boldsymbol{a}_{\mu}, \boldsymbol{\beta}_{\mu}, \mu=0,1$, and

$S_{\text {deph } \boldsymbol{\beta}}=\boldsymbol{a}_{0} \cdot \boldsymbol{\beta}\left(\mathbf{\gamma}_{0} \cdot \boldsymbol{\beta}-\mathbf{\gamma}_{1} \cdot \boldsymbol{\beta}\right)+\boldsymbol{a}_{1} \cdot \boldsymbol{\beta}\left(\mathbf{\gamma}_{0} \cdot \boldsymbol{\beta}+\mathbf{\gamma}_{1} \cdot \boldsymbol{\beta}\right)$

is the CHSH value for strong $\boldsymbol{a}_{\mu}, \boldsymbol{\gamma}_{\mu}$ measurements, but linked by a dephasing channel (i.e., a strong measurement whose outcome is discarded) along direction $\boldsymbol{\beta}$. 
Generalized monogamy condition

Substituting the maximal values $S_{\text {strong }} \rightarrow 2 \sqrt{2}$ and $S_{\text {deph }} \rightarrow 2$ in Eqs. (8)(10), and finding $k$ that gives them equal value, we obtain:

$S_{\max } \approx 2.426$

Note that any evolution between measurements other than identity cannot increase the maximal value achievable by $S_{\text {strong }}$ and $S_{\text {deph }}$ (for any $\boldsymbol{\beta})$, thus the above upper bound remains valid for an arbitrary Markov process and represents a generalized monogamy relation for three temporally separated measurements in the configuration considered in this work.

Although the above upper bound is probably not tight, it indicates that even with genuine multipartite temporal correlations revealed by weak measurements, it is not possible to reach the algebraic maximal simultaneous violation of the CHSH inequality for all pairs. Indeed, the algebraic joint CHSH maximum is $10 / 3>2.426$, which can be seen as follows: A deterministic outcome assignment can achieve maximal violation, $S=4$, for only two out of three pairs. This is because there are two combinations of settings, $(x, y, z)=(0,1,0),(1,0,1)$, for which the maximal violation would require conflicting measurement outcomes, $A=$ $C=B=-A$ for $(0,1,0)$ and $C=A=B=-C$ for $(1,0,1)$. This means that a deterministic probability distribution which achieves $S=4$ for two pairs will be bounded by $S=2$ for the third. In order to achieve an equal CHSH value for the three pairs, we have to take an equal mixture of three distributions, where one of the three pairs in turn is limited by the classical bound. This produces, on average, the value $(4+4+2) / 3=10 / 3$ for each pair. We finally note that the algebraic upper bound can be reached by non-quantum correlations that satisfy causality, namely, where future settings are not correlated to past outcomes. Indeed, we can construct the desired deterministic vertices (with two maximal CHSH values and one at the classical bound) by first setting all of Alice's outcomes (e.g., all to $A=1$ ), then choosing the outcomes of $B$ as a function of $x$ and $y$ (but not of $z$ ) to get either $S^{A B}=4$ or $S^{A B}=2$, and finally choosing the outcomes for $C$, to reach $S^{A C}=S^{B C}=4$ (if we had $S^{A B}=2$ ), or the algebraic maximum for one of the two $\mathrm{CHSH}$ values and the classical bound for the other (if we had $\left.S^{\mathrm{AB}}=4\right)$.

Data and code availability

All relevant data and code are available from the authors upon reasonable request.

\section{ACKNOWLEDGEMENTS}

We thank T. Vulpecula for experimental assistance. This work was supported in part by the Centres for Engineered Quantum Systems (EQUS, CE110001013, CE170100009) and for Quantum Computation and Communication Technology (CE110001027) and the Engineering and Physical Sciences Research Council (grant number EP/N002962/1). F.C. acknowledges support through an Australian Research Council Discovery Early Career Researcher Award (DE170100712), A.G.W. through a UQ Vice-Chancellor's Senior Research and Teaching Fellowship. M.E.G. would like to thank the University of Queensland for support during his sabbatical visit. This publication was made possible through the support of a grant from the John Templeton Foundation. The opinions expressed in this publication are those of the authors and do not necessarily reflect the views of the John Templeton Foundation. We acknowledge the traditional owners of the land on which the University of Queensland is situated, the Turrbal and Jagera people.

\section{AUTHOR CONTRIBUTIONS}

M.R., A.F., A.G.W. conceived and designed the experiment. M.R. and M.E.G. performed the experiment, collected, and analyzed the data. F.C. derived the theory results. All authors contributed to writing the manuscript.

\section{ADDITIONAL INFORMATION}

Competing interests: The authors declare no competing interests.

Publisher's note: Springer Nature remains neutral with regard to jurisdictional claims in published maps and institutional affiliations.

\section{REFERENCES}

1. Leggett, A. J. \& Garg, A. Quantum mechanics versus macroscopic realism: is the flux there when nobody looks?. Phys. Rev. Lett. 54, 857 (1985).

2. Markiewicz, M. et al. Unified approach to contextuality, nonlocality, and temporal correlations. Phys. Rev. A 89, 042109 (2014).

3. Brukner, C., Taylor, S., Cheung, S. \& Vedral, V. Quantum entanglement in time. Preprint at https://arxiv.org/abs/quant-ph/0402127 (2004).

4. Żukowski, M. Temporal inequalities for sequential multi-time actions in quantum information processing. Front. Phys. 9, 629-633 (2014).

5. Markiewicz, M., Przysiężna, A., Brierley, S. \& Paterek, T. Genuinely multipoint temporal quantum correlations and universal measurement-based quantum computing. Phys. Rev. A 89, 062319 (2014).

6. Chen, Y-N. et al. Temporal steering inequality. Phys. Rev. A 89, 032112 (2014).

7. Costa, F., Ringbauer, M., Goggin, M. E., White, A. G. \& Fedrizzi, A. A unifying framework for spatial and temporal quantum correlations. Preprint at https:// arxiv.org/abs/1710.01776 (2017).

8. Coffman, V., Kundu, J. \& Wootters, WK. Distributed entanglement. Phys. Rev. A 61 052306 (2000).

9. Terhal, B. M. Is entanglement monogamous?. IBM J. Res. Dev. 48, 71-78 (2004).

10. Bell, J. S. On the Einstein Podolsky Rosen paradox. Physics 1, 195-200 (1964).

11. Clauser, J., Horne, M., Shimony, A. \& Holt, R. Proposed experiment to test local hidden-variable theories. Phys. Rev. Lett. 23, 880-884 (1969).

12. Streltsov, A., Adesso, G., Piani, M. \& Bruß, D. Are general quantum correlations monogamous?. Phys. Rev. Lett. 109, 050503 (2012).

13. Toner, B. Monogamy of non-local quantum correlations. Proc. R. Soc. A 465, 59-69 (2009).

14. Pawłowski, M. Security proof for cryptographic protocols based only on the monogamy of Bell's inequality violations. Phys. Rev. A 82, 032313 (2010).

15. Oreshkov, O., Costa, F. \& Brukner, Č. Quantum correlations with no causal order. Nat. Commun. 3, 1092 (2012).

16. Kretschmann, D. \& Werner, R. F. Quantum channels with memory. Phys. Rev. A 72, 062323 (2005).

17. Gutoski, G. \& Watrous, J. Toward a general theory of quantum games. In Proc. of 39th ACM STOC, Association for Computing Machinery (ACM), New York, NY, USA. 565-574 (2006).

18. Aharonov, Y., Popescu, S., Tollaksen, J. \& Vaidman, L. Multiple-time states and multiple-time measurements in quantum mechanics. Phys. Rev. A 79, 052110 (2009).

19. Cotler, J., Jian, C.-M., Qi, X.-L. \& Wilczek, F. Superdensity operators for spacetime quantum mechanics. Preprint at https://arxiv.org/abs/1711.03119 (2017).

20. Pollock, F. A., Rodríguez-Rosario, C., Frauenheim, T., Paternostro, M. \& Modi, K Non-Markovian quantum processes: complete framework and efficient characterization. Phys. Rev. A 97, 012127 (2018).

21. Leifer, M. \& Spekkens, R. W. Towards a formulation of quantum theory as a causally neutral theory of Bayesian inference. Phys. Rev. A 88, 052130 (2013).

22. Fitzsimons, J. F., Jones, J. A. \& Vedral, V. Quantum correlations which imply causation. Sci. Rep. 5, 18281 (2015).

23. Zhao, Z. et al. Geometry of quantum correlations in space-time. Preprint at https://arxiv.org/abs/1711.05955 (2017).

24. Horsman, D., Heunen, C., Pusey, M. F., Barrett, J. \& Spekkens, R. W. Can a quantum state over time resemble a quantum state at a single time?. Proc. R. Soc. A 473 20170395 (2017).

25. Davies, E. B. \& Lewis, J. T. An operational approach to quantum probability. Comm. Math. Phys. 17, 239-260 (1970).

26. Chiribella, G., D'Ariano, G. M. \& Perinotti, P. Quantum circuit architecture. Phys. Rev. Lett. 101, 060401 (2008).

27. Chiribella, G., D'Ariano, G. \& Perinotti, P. Theoretical framework for quantum networks. Phys. Rev. A 80, 022339 (2009).

28. Costa, F. \& Shrapnel, S. Quantum causal modelling. New J. Phys. 18, 063032 (2016).

29. Branciard, C., Rosset, D., Gisin, N. \& Pironio, S. Bilocal versus nonbilocal correlations in entanglement-swapping experiments. Phys. Rev. A 85(3), 032119 (2012).

30. Braunstein, S. L. \& Mann, A. Measurement of the Bell operator and quantum teleportation. Phys. Rev. A 51, R1727-R1730 (1995).

31. Langford, N. et al. Demonstration of a simple entangling optical gate and its use in Bell-state analysis. Phys. Rev. Lett. 95, 210504 (2005)

32. Fedrizzi, A., Almeida, M. P., Broome, M. A., White, A. G. \& Barbieri, M. Hardy's paradox and violation of a state-independent Bell inequality in time. Phys. Rev. Lett. 106, 200402 (2011).

33. Ringbauer, $M$. et al. Experimental joint quantum measurements with minimum uncertainty. Phys. Rev. Lett. 112, 020401 (2014).

34. Horodecki, R., Horodecki, M. \& Horodecki, K. Quantum entanglement. Rev. Mod. Phys. 81, 865-942 (2009).

35. Kleinmann, M., Gühne, O., Portillo, J. R., Larsson, J.-Å. \& Cabello, A. Memory cost of quantum contextuality. New J. Phys. 13, 113011 (2011). 
36. Ringbauer, M. \& Chaves, R. Probing the non-classicality of temporal correlations. Quantum 1, 35 (2017)

37. Das, S., Aravinda, S., Srikanth, R. \& Home, D. Unification of Bell, Leggett-Garg and Kochen-Specker inequalities: hybrid spatio-temporal inequalities. Eur. Phys. Lett. 104, 60006 (2013).

38. Chen, S.-L. et al. Spatio-temporal steering for testing nonclassical correlations in quantum networks. Sci. Rep. 7, 3728 (2017).

39. Xu, Z.-P. \& Cabello, A. Quantum correlations with a gap between the sequential and spatial cases. Phys. Rev. A 96, 012122 (2017).

Open Access This article is licensed under a Creative Commons Attribution 4.0 International License, which permits use, sharing, adaptation, distribution and reproduction in any medium or format, as long as you give appropriate credit to the original author(s) and the source, provide a link to the Creative Commons license, and indicate if changes were made. The images or other third party material in this article are included in the article's Creative Commons license, unless indicated otherwise in a credit line to the material. If material is not included in the article's Creative Commons license and your intended use is not permitted by statutory regulation or exceeds the permitted use, you will need to obtain permission directly from the copyright holder. To view a copy of this license, visit http://creativecommons. org/licenses/by/4.0/.

(c) The Author(s) 2018 